\title{
Techniques for Self-Checking Combinational Logic Synthesis
}

\author{
FADI BUSABA and PARAG K. LALA \\ Department of Electrical Engineering, North Carolina A\&T State University, Greensboro, NC 27411
}

(Received October 12, 1992; Revised March 15, 1993)

\begin{abstract}
This paper presents techniques for designing arbitrary combinational circuits so that any single stuck-at fault will result in either single bit error or unidirectional multibit error at the output. If the outputs are encoded using Berger code or m-out-of-n code, then the proposed technique will enable on-line detection of faults in the circuit. An algorithm for indicating whether a certain fault at an input will create bidirectional error at the output is presented. An input encoding algorithm and an output encoding algorithm that ensure that every fault will either produce single bit error or unidirectional multibit error at the output are proposed. If there are no input fault which produces bidirectional error, no internal stuck-at fault will result in such an error irrespective of the way the circuit is implemented. Thus, only single bit or unidirectional multibit error will result in the presence of a fault in the circuit. The proposed techniques have been applied to MCNC benchmark circuits and the overhead is estimated.
\end{abstract}

Key Words: Error-detection, Self-checking, Logic synthesis, Unidirectional errors, Input encoding, Output encoding

\section{INTRODUCTION}

$\mathbf{W}$ ith the increase in the complexity and density of VLSI chips, transient/intermittent faults have emerged as the dominant failure modes in VLSI circuits [1-2]. Conventional off-line testing schemes do not detect transient faults since the detection of these faults requires continuous monitoring of the outputs; i.e. the circuits have to be self-checking. A self-checking circuit usually consists of a functional block that generates the encoded outputs, and a checker that checks the validity of the outputs [35]. Self-checking circuits that use m-out-of-n code [6] or Berger code [7] for output encoding, detect stuckat faults that cause single bit error or unidirectional multibit error. Designing logic circuits such that any stuck at-fault causes single bit error or unidirectional multibit error is a challenging problem. Previous work either use PLA structure, or perform algebraic factorization for two-level networks by restricting the use of inverters at the inputs and using only AND/ OR gates [8-9]. Also, it is assumed that all input lines and their inversions are fault free. In [14], monotone functions and inverter free realization were used to design strongly fault secure logic net- works. Similarly, inverter free realizations were used in [15] for the design of strongly fault secure and strongly code disjoint circuits. The restriction on the type of gates and on synthesis procedures used for logic circuits usually increases the area overhead. In this paper, we propose techniques for designing bidirectional error-free combinational circuits based on input encoding and output encoding schemes that do not restrict the way the circuit is implemented. The output encoding strategy does not form codewords as used in conventional coding techniques. Our intention here is to design the functional part of a self-checking circuit such that any single stuckat fault will create either single bit error or unidirectional multibit error. Thus by incorporating additional check bits, the output of the functional block can be designed to be a single and unidirectional error detecting code; e.g., Berger code and m-outof-n code.

The techniques presented in this paper can be directly applied to logic circuits described in the PLA format, where symbolic representation is used for the inputs or the outputs or both; i.e., the inputs or the outputs have not been assigned any binary codes. The aim of this paper is to encode the inputs or the 
outputs so that any single stuck-at fault can only create single bit error or unidirectional multibit error at the output. Thus, these techniques will be useful only if self-checking is considered at the design level i.e., before the circuit is actually implemented.

Preliminaries and definition are given in section 2 . In sections 3 , an algorithm for detection of faults that might cause bidirectional error at the outputs is presented. An input encoding algorithm and an output encoding algorithm are presented in section 4. A detailed example is given at the end of section 4 where the input and output encoding algorithm are applied to a benchmark circuit. A VLSI implementation of each technique is provided, and the area overhead is estimated. The proposed techniques have been applied to MCNC benchmark circuits, and the results are reported in section 5 .

\section{PRELIMINARIES}

Before presenting the proposed algorithms, we need to consider the following definitions.

Definition 1: A variable is a symbol representing a single coordinate of the boolean space (e.g. $a$ ).

Definition 2: A literal is a variable or its negation (e.g. $a$ or $a^{\prime}$ ).

Definition 3: A cube is a set $\mathrm{C}$ of literals such that if $x$ belongs to $\mathrm{C}$, then $x^{\prime}$ does not belong to $\mathrm{C}$.

Definition 4: A minterm is a cube with only 0 and 1 entries.

Definition 5: A function is unate if in its minimal sum-of-products expression each variable appears either in a complemented form or in an uncomplemented form but not in both.

Definition 6: The $\operatorname{dist}\left(\mathrm{C}_{1}, \mathrm{C}_{2}\right)$, where $\mathrm{C}_{1}$ and $\mathrm{C}_{2}$ are different input cubes, is the number of bit positions in which they differ when these bit positions are either 1's or a 0's, but not don't cares. For example $\operatorname{dist}\left(\mathrm{C}_{1}, \mathrm{C}_{2}\right)$, where $\mathrm{C}_{1}(\mathrm{abcd})=1010$ and $\mathrm{C}_{2}(\mathrm{abcd})$ $=-110$, equals to one because the two input cubes differs only in position $b$.

Definition 7: Two output vectors $\mathrm{O}_{1}$ and $\mathrm{O}_{2}$ are partially bidirectional if there exist at least two outputs bits which are 10 in $\mathrm{O}_{1}$ and 01 in $\mathrm{O}_{2}$ or vice versa. For example, $\mathrm{O}_{1}=1100$ and $\mathrm{O}_{2}=0101$ are partially bidirectional whereas $\mathrm{O}_{1}=1000$ and $\mathrm{O}_{2}=1111$ are not.

Definition 8: Two output vectors $\mathrm{O}_{\mathrm{i}}$ and $\mathrm{O}_{\mathrm{j}}$ are adjacent if $\operatorname{dist}\left(C_{i}, C_{j}\right)=1$, where $C_{i}$ and $C_{j}$ are the input cubes corresponding to $\mathrm{O}_{\mathrm{i}}$ and $\mathrm{O}_{\mathrm{j}}$ respectively. Definition 9: Two input cubes $\mathrm{C}_{\mathrm{i}}$ and $\mathrm{C}_{\mathrm{j}}$ are bidirectionally adjacent if their corresponding outputs, $\mathrm{O}_{\mathrm{i}}$ and $\mathrm{O}_{\mathrm{j}}$, are partially bidirectional.
Definition 10: Two input cubes are called $\boldsymbol{m}$-bidirectional if they only differ in position $m$ and their corresponding outputs are partially bidirectional. For example, input cubes $\mathrm{C}_{1}$ and $\mathrm{C}_{3}$ in Table $\mathrm{I}$ are $a$ bidirectional.

Definition 11: Bidirectionality set of two bidirectionally adjacent cubes is the set that contains all the variables in which the two cubes differ. For example, the bidirectionality set of $\mathrm{C}_{1}$ and $\mathrm{C}_{2}$ in Table $\mathrm{I}$ is $\{b$, $c$ s since they differ in positions $b$ and $c$.

Definition 12: The num_inv(n1, n2) is the number of inversions on the path from $\mathrm{n} 1$ to $\mathrm{n} 2$ modulo 2 , where $\mathrm{n} 1$ and $\mathrm{n} 2$ are two nodes inside a digital circuit.

Definition 13: A fault $f$ creates a unidirectional error if the correct and the faulty outputs are not partially bidirectional.

Definition 14: An undirected graph $G$ containing the set of vertices $\mathrm{V}$ and the set of edges $\mathrm{E}$ is denoted by $G(V, E) \cdot G^{\prime}\left(V^{\prime}, E^{\prime}\right)$ is a subgraph of $G$ if $V^{\prime}$ is a subset of $\mathrm{V}$, and an edge joins two vertices in $\mathrm{G}^{\prime}$ if an edge joins the same two vertices in $G$. A fully connected subgraph of $G$ is a graph $G_{s}\left(V_{s}, E_{s}\right)$ such that $V_{s}$ is subset of $V$, and any two vertices in $G_{s}$ are connected by an edge. A graph $G_{1}\left(V_{1}, E_{1}\right)$ covers graph $G_{2}\left(V_{2}, E_{2}\right)$ if the following are satisfied:

(i) the number of vertices in $E_{1}$, is equal to or greater than the number of vertices in $E_{2}$.

(ii) if there exists an edge between two nodes in $\mathrm{V}_{2}$, there should be also an edge between the same two nodes in $\mathrm{V}_{1}$.

Definition 15: A graph, $\mathrm{G}_{\mathrm{m}}$, containing $2^{\mathrm{m}}$ vertices is unidirectional graph iff

(i) each vertex is uniquely represented by an mbit number.

(ii) any two vertices are connected if they are not partially bidirectional.

The following definitions are taken from [12].

Definition 16: A controlling value at a gate input is the value that determines the value at the output of the gate independent of the other inputs. For example, 0 is a controlling value for an AND gate. A noncontrolling value at a gate input has no effect on

TABLE I

Truth Table of a Logic Circuit

\begin{tabular}{ccccccccc}
\hline & \multicolumn{4}{c}{ inputs } & \multicolumn{3}{c}{ Symbolic } & \multicolumn{3}{c}{ outputs } \\
cubes & $\mathrm{a}$ & $\mathrm{b}$ & $\mathrm{c}$ & $\mathrm{d}$ & outputs & $\mathrm{O}_{1}$ & $\mathrm{O}_{2}$ & $\mathrm{O}_{3}$ \\
\hline $\mathrm{C}_{1}$ & 0 & 1 & 0 & 1 & $\mathrm{Z}_{1}$ & 1 & 0 & 0 \\
$\mathrm{C}_{2}$ & 0 & 0 & 1 & 1 & $\mathrm{Z}_{2}$ & 0 & 1 & 0 \\
$\mathrm{C}_{3}$ & 1 & - & 0 & 1 & $\mathrm{Z}_{2}$ & 0 & 1 & 0 \\
\hline
\end{tabular}


the output of the gate. For example, 1 is a noncontrolling value for an AND gate.

Definition 17: A path is in a combinational circuit consists of connections and gates, where connection $i$ connects gate $(i-1)$ and gate $i$. In other words, an input to gate $i$ is the output from gate $(i-1)$. The inputs to a gate $i$ other than the output from gate $(i-1)$ are called side inputs.

Definition 18: A path is said to be statistically sensitizable if there exists an input cube that sets all side inputs to noncontrolling values.

Definition 19: An event is the transition from 0(1) to $1(0)$.

Definition 20: A primitigve gate is prime is none of its inputs can be removed without causing the resulting circuit to be functionally different. A gate is irredundant if its removal causes the resulting circuit to be functionally different. A gate-level circuit is said to be prime if all the gates are prime and irredundant if all the gates are irredundant. A gate-level circuit is prime and irredundant if and only if it is $100 \%$ testable for all single stuck-at faults [13].

\section{DETECTION OF BIDIRECTIONAL FAULTS AT INPUT LINES}

A fault at a node $f$ results in unidirectional error at the outputs if the number of inversions from the fault site to the outputs is the same; in other words, num -inv $\left(f, o_{i}\right)$ is the same, where $o_{i}$ is an input line that is affected by $f$.

The following two lemmas identify the existence of bidirectional error due to a fault on an input line, and how to eliminate such errors.

Lemma 1: If there exist two $x$-bidirectional input cubes, then a fault at input $x$ may create a bidirectional error at the output.

Proof: Suppose cubes $\mathrm{C}_{1}$ and $\mathrm{C}_{2}$ are $x$-bidirectional, then $\mathrm{C}_{1}$ and $\mathrm{C}_{2}$ differ only in variable $x$ and the outputs corresponding to $\mathrm{C}_{1}$ and $\mathrm{C}_{2}$ i.e., $\mathrm{O}_{1}$ and $\mathrm{O}_{2}$, are partially bidirectional. Therefore, if $\mathrm{C}_{1}$ is activated, a fault at input $x$ may activate $\mathrm{C}_{2}$ instead of $\mathrm{C}_{1}$ producing $\mathrm{O}_{2}$ instead of $\mathrm{O}_{1}$, thus creating a bidirectional error. Q.E.D.

Lemma 2: If no two input cubes are $x$-bidirectional, than a fault at an input line will always produce a unidirectional error.

Proof: If no $x$-bidirectional input cubes exist, then either (1) the distance between all bidirectionally adjacent input cubes is zero or greater than or equal to two, or (2) any adjacent outputs are not partially
TABLE II

Specification of a Full Adder

\begin{tabular}{cccccc}
\hline cubes & $\mathrm{A}$ & $\mathrm{B}$ & $\mathrm{C}$ & $\mathrm{O}_{1}$ & $\mathrm{O}_{2}$ \\
\hline $\mathrm{C}_{1}$ & 1 & 0 & 0 & 1 & 0 \\
$\mathrm{C}_{2}$ & 0 & 1 & 0 & 1 & 0 \\
$\mathrm{C}_{3}$ & 0 & 0 & 1 & 1 & 0 \\
$\mathrm{C}_{4}$ & 1 & 1 & 1 & 1 & 1 \\
$\mathrm{C}_{5}$ & 1 & 1 & 0 & 0 & 1 \\
$\mathrm{C}_{6}$ & 0 & 1 & 1 & 0 & 1 \\
$\mathrm{C}_{7}$ & 1 & 0 & 1 & 0 & 1 \\
$\mathrm{C}_{8}$ & 0 & 0 & 0 & 0 & 0 \\
\hline
\end{tabular}

bidirectional. For case (1), no fault at an input line will activate cube $C_{j}$ instead of $C_{i}$ where $C_{i}$ and $C_{j}$ are $m$-bidirectional; thus every fault results in unidirectional error at the output. For case (2), a fault at the input may activate $C_{j}$ instead of $C_{i}$. Since the outputs corresponding to $C_{i}$ and $C_{j}$ are not partially bidirectional, the fault results in unidirectional error at the output. Q.E.D.

The following algorithm identifies which faults at the input lines might cause bidirectional error at the output.

\section{Algorithm 1:}

1. For every possible output $\mathrm{O}_{\mathrm{i}}$ in a circuit, group all other outputs that are partially bidirectional with $\mathrm{O}_{\mathrm{i}}$.

2. For an output $\mathrm{O}_{\mathrm{i}}$ in the group, find $\operatorname{dist}\left(\mathrm{C}_{\mathrm{i}}, \mathrm{C}_{\mathrm{i}}\right)$ where $\mathrm{C}_{\mathrm{i}}$ is the cube corresponding to $\mathrm{O}_{i}$, and $\mathrm{C}_{\mathrm{j}}$ is a cube corresponding to $\mathrm{O}_{\mathrm{j}}$.

3. If $\operatorname{dist}\left(C_{i}, C_{j}\right)$ equals to 1 and the two cubes differ in variable $x$, then add $\left(\mathrm{C}_{\mathrm{i}}, \mathrm{C}_{\mathrm{j}}, x\right)$ to the set of possible bidirectional faults.

To illustrate the application of the algorithm, let us consider the truth Table description of a Full Adder shown in Table II.

The first step results in the following group of outputs $(10,01)$. By applying step 2 and step 3 , the following set is obtained: $\left\{\left(C_{1}, C_{5}, B\right),\left(C_{1}, C_{7}, C\right)\right.$, $\left.\left(\mathrm{C}_{2}, \mathrm{C}_{5}, \mathrm{~A}\right),\left(\mathrm{C}_{2}, \mathrm{C}_{6}, \mathrm{C}\right),\left(\mathrm{C}_{3}, \mathrm{C}_{6}, \mathrm{~B}\right),\left(\mathrm{C}_{3}, \mathrm{C}_{7}, \mathrm{~A}\right)\right\}$ The element $\left(\mathrm{C}_{1}, \mathrm{C}_{5}, \mathrm{~B}\right)$ indicates that cubes $\mathrm{C}_{1}$ and $\mathrm{C}_{5}$ are $\mathrm{B}$-bidirectional; i.e., if $\mathrm{C}_{1}$ is applied to the circuit and input $B$ gets stuck-at-1, then the input will correspond to $\mathrm{C}_{5}$, which will result in the output 01 instead of the fault free output 10 .

\section{TECHNIQUES FOR BIDIRECTIONAL ERROR ELIMINATION}

Preventing faults at the input lines of a circuit from causing bidirectional error at the output can be guar- 
anteed by 'properly' encoding the inputs or the outputs. The input or output encoding should be done such that no two input cubes become $m$-bidirectional, thus eliminating the possibility of bidirectional error at the output. (Lemma 2). We will first describe the input encoding strategy.

\subsection{Input Encoding}

The input encoding strategy assigns codes to two bidirectionally adjacent inputs cubes, $\mathrm{C}_{\mathrm{i}}$ and $\mathrm{C}_{\mathrm{j}}$, such that $\operatorname{dist}\left(\mathrm{C}_{\mathrm{i}}, \mathrm{C}_{\mathrm{j}}\right)$ is either $\geq 2$, or equal to 0 . We consider the two cases separately.

Case 1: In this case, $C_{i}$ and $C_{j}$ are coded such that $\operatorname{dist}\left(\mathrm{C}_{\mathrm{i}}, \mathrm{C}_{\mathrm{j}}\right) \geq 2$. Consider a digital circuit with $\mathrm{I}$ different input symbols and $\mathrm{O}$ encoded outputs. The steps required to satisfy the distance requirement are:

1. For each possible input cube in a circuit, group all other input cubes that are bidirectionally adjacent.

2. Assign codes with distance greater than or equal to 2 for any bidirectional adjacent input cubes by using minimum number of encoding bits.

3. If the assignment is not possible, increase the number of encoding bits by one and repeat step 2.

Consider the example shown in Table III in which each input cube is bidirectionally adjacent with four other cubes. Steps 1 and 2 suggest that cubes $C_{1}, C_{2}$, $\mathrm{C}_{3}$ and $\mathrm{C}_{4}$ have to be at a distance greater than or equal to two from cubes $\mathrm{C}_{5}, \mathrm{C}_{6}, \mathrm{C}_{7}$ and $\mathrm{C}_{8}$. This can be illustrated with a graph (Figure 1) which is constructed with input cubes as vertices, and with an edge connecting two vertices if they are bidirectionally adjacent. Thus the distance should be greater than or equal to 2 for any vertices joined by an edge. If minimum number of bits for encoding is used and

TABLE III

Description of a Logic Circuit

\begin{tabular}{ccc}
\hline $\begin{array}{c}\text { Input } \\
\text { Cubes }\end{array}$ & $\mathrm{O}_{1}$ & Outputs \\
\hline $\mathrm{C}_{1}$ & 1 & $\mathrm{O}_{2}$ \\
$\mathrm{C}_{2}$ & 1 & 0 \\
$\mathrm{C}_{3}$ & 1 & 0 \\
$\mathrm{C}_{4}$ & 1 & 0 \\
$\mathrm{C}_{5}$ & 0 & 0 \\
$\mathrm{C}_{6}$ & 0 & 1 \\
$\mathrm{C}_{7}$ & 0 & 1 \\
$\mathrm{C}_{8}$ & 0 & 1 \\
\hline
\end{tabular}

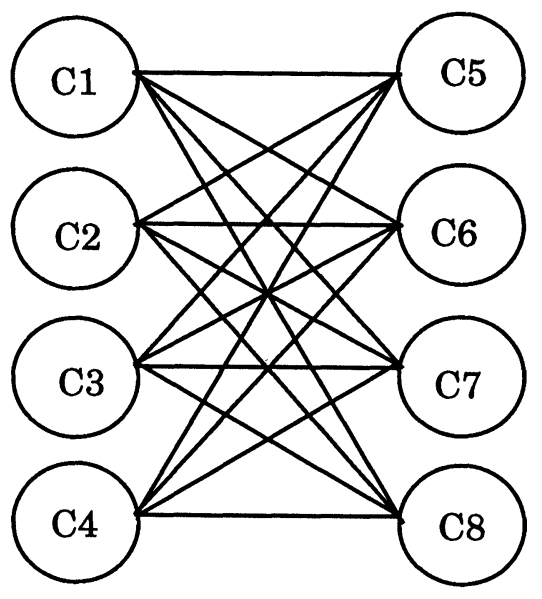

FIGURE 1

$\mathrm{C}_{1}$ is assigned to 000 , then cubes $\mathrm{C}_{5}, \mathrm{C}_{6}, \mathrm{C}_{7}$ and $\mathrm{C}_{8}$ have to be coded as 101, 110, 011 and 111 respectively. Consequently, any code for $\mathrm{C}_{2}, \mathrm{C}_{3}$ or $\mathrm{C}_{4}$ will have a distance of one from their bidirectionally adjacent cubes which conflicts with the constraint in step 2. If four bits are used to encode the input, one possible input coding is:

$$
\begin{array}{ll}
0000 \longrightarrow \mathrm{C}_{1} & 0010 \longrightarrow \mathrm{C}_{2} \\
0100 \longrightarrow \mathrm{C}_{3} & 0110 \longrightarrow \mathrm{C}_{4} \\
1101 \longrightarrow \mathrm{C}_{5} & 1111 \longrightarrow \mathrm{C}_{6} \\
1001 \longrightarrow \mathrm{C}_{7} & 1011 \longrightarrow \mathrm{C}_{8}
\end{array}
$$

If the cubes are encoded in this manner, cubes $\mathrm{C} 1$, $\mathrm{C} 2, \mathrm{C} 3$ and $\mathrm{C} 4$ can be reduced to the cube $0--0$, and similarly cubes $\mathrm{C} 5, \mathrm{C} 6, \mathrm{C} 7$, and $\mathrm{C} 8$ can be reduced to 1--1. Therefore, any fault at the input will result in unidirectional error at the output.

An alternative approach for input encoding is to use m-out-of-n codes since the minimum distance between any two codes is two. In addition, they are easily implemented without significant increase in the number of input lines.

Case 2: In this case, $\mathrm{C}_{\mathrm{i}}$ and $\mathrm{C}_{\mathrm{j}}$ are coded such that $\operatorname{dist}\left(\mathrm{C}_{\mathrm{i}}, \mathrm{C}_{\mathrm{j}}\right)=0$. Let us assume two $x$-bidirectional input cubes, $\mathrm{C}_{\mathrm{i}}$ and $\mathrm{C}_{\mathrm{j}}$, where the variable $x$ is 0 in $\mathrm{C}_{\mathrm{i}}$ and 1 in $\mathrm{C}_{\mathrm{j}}$. Encoding the input by adding one more line for the variable $x$ such that it is represented by -0 in $C_{i}$ and by $1-$ in $C_{j}$ will make $\operatorname{dist}\left(C_{i}, C_{j}\right)=$ 0 . In the proposed input encoding strategy, a 0 and a 1 in the input cubes are replaced by -0 and 1 respectively. Consider for example two $a$-bidirec- 
tional input cubes $\mathrm{C}_{1}$ (abcd) $=1010$ and $\mathrm{C}_{2}$ (abcd) $=0010$. Since $a$ is a 1 in $C_{1}$ and 0 in $C_{2}$, the new input encoding, $\mathrm{C}_{1}\left(\mathrm{aa} \mathrm{a}_{1} \mathrm{bcd}\right)=1-010$ and $\mathrm{C}_{2}\left(\mathrm{aa}_{1} \mathrm{bcd}\right)$ $=-0010$, will guarantee that $\operatorname{dist}\left(\mathrm{C}_{1}, \mathrm{C}_{2}\right)$ is zero, and thus the fault at input $a$ that might cause bidirectional error at the ouput is eliminated. It is important to note that input variable $a\left(a_{1}\right)$ appears either as a don't care '-' or as a $1(0)$ in the input cubes. If for every input variable $x$, there exist at least two $x$ bidirectional input cubes, the number of input lines will be doubled because $x$ will be replaced by two inputs $x$ and $x_{1}$. Thus, in the worst case, this strategy doubles the number of input lines. In such a situation, every input variable will be either present as a 0 or 1 but not both in the input cubes.

In addition, this encoding process may result in $x$ bidirectional input cubes even in the absence of $x$ bidirectional input cubes in the original specification. For example, in Table $\mathrm{I}, \mathrm{C}_{1}$ and $\mathrm{C}_{3}$ are $a$-bidirectional, and cubes $C_{1}$ and $C_{2}$ are bidirectionally adjacent with their corresponding bidirectionality set $\{b, c\}$. To make $\operatorname{dist}\left(C_{1}, C_{3}\right)$ zero, one more input variable is added such that $C_{1}=-0101, C_{2}=-0011$ and $\mathrm{C}_{3}=1--01$. In this case, cubes $\mathrm{C}_{1}$ and $\mathrm{C}_{2}$ become $c$-bidirectional; that is because the bidirectionality set of $\mathrm{C}_{1}$ and $\mathrm{C}_{2}$ was $\{\mathrm{a}, \mathrm{c}\}$ and by replacing $a$ with -0 and 1- in $\mathrm{C}_{1}$ and $\mathrm{C}_{2}$ respectively, variable $a$ can be taken out from the set.

The input encoding algorithm proposed below satisfies the distance requirement.

\section{Algorithm 1:}

Repeat

For every input cube $\mathrm{Ci}$

For every input cube $\mathrm{Cj}$

If $\mathrm{Ci}$ and $\mathrm{Cj}$ are $x$-bidirectional

\{

increase the number of input encoding bits by one as follows:

substitute in all cubes the location of $x$ by

1 - if it is a 1 , by 0 - if it is a 0 and by -- if it

is a - (don't care).

\}

\} until there is no $x$-bidirectional cubes.

After applying algorithm 1 to the circuit description of Table II, another circuit description, Table IV, is derived with different input encoding.

The new input encoding doubles the number of variables but keeps the number of literals unchanged. In this case, the output equations are:

$$
\begin{aligned}
& O^{1}=A \bar{B}^{1} \bar{C}^{1}+\bar{A}^{1} B \bar{C}^{1}+\bar{A}^{1} \bar{B}^{1} C \\
& O_{2}=A B+B C+A C
\end{aligned}
$$

TABLE IV

Specification FA After Input Encoding

\begin{tabular}{ccccccccc}
\hline cubes & $\mathrm{A}$ & $\mathrm{A}_{1}$ & $\mathrm{~B}$ & $\mathrm{~B}_{1}$ & $\mathrm{C}$ & $\mathrm{C}_{1}$ & $\mathrm{O}_{1}$ & $\mathrm{O}_{2}$ \\
\hline $\mathrm{C}_{1}$ & 1 & - & - & 0 & - & 0 & 1 & 0 \\
$\mathrm{C}_{2}$ & - & 0 & 1 & - & - & 0 & 1 & 0 \\
$\mathrm{C}_{3}$ & - & 0 & - & 0 & 1 & - & 1 & 0 \\
$\mathrm{C}_{4}$ & 1 & - & 1 & - & 1 & - & 1 & 1 \\
$\mathrm{C}_{5}$ & 1 & - & 1 & - & - & 0 & 0 & 1 \\
$\mathrm{C}_{6}$ & - & 0 & 1 & - & 1 & - & 0 & 1 \\
$\mathrm{C}_{7}$ & 1 & - & - & 0 & 1 & - & 0 & 1 \\
$\mathrm{C}_{8}$ & - & 0 & - & 0 & - & 0 & 0 & 0 \\
\hline
\end{tabular}

These output functions are unate since the variables either appear in their complemented or uncomplemented form but not both.

Lemma 3: If the minimized output functions are unate, every fault (internal or at the input lines) will cause either single bit error or unidirectional multibit error at the output regardless of the way the circuit is implemented.

Proof: If the outputs functions are unate, variables appear in either complemented or non-complemented form but not both. Thus, if a variable appears in a non-complemented form in a function, the variable either does not get inverted or go through even number of inversions from the input to the output. Similarly, if a variable appears in complemented form, it either does not get inverted or goes through odd number of inversions. Therefore, if a variable is in uncomplemented (complemented) form, and a fault occurs at the corresponding input line, this fault will create unidirectional error since there is even (odd) number of inversions from the input line to the outputs. On the other hand, if a fault exists at node $x$ and affects k outputs, then, there will at least $\mathrm{k}$ different paths $\mathrm{P} 1$ to $\mathrm{Pk}$ from the fault site to the $\mathrm{k}$ affected outputs. Suppose node $x$ is affected by one of the inputs, say $a$ (Figure 2). Since the number of inversions (modulo 2) between input $a$ and the outputs is the same,

$\operatorname{num} \_\operatorname{inv}\left(a, o_{1}\right)=$ num_inv $\left(a, o_{2}\right)=. .=\operatorname{num} \_i n v(a$, $\left.o_{k}\right)$. In other words,

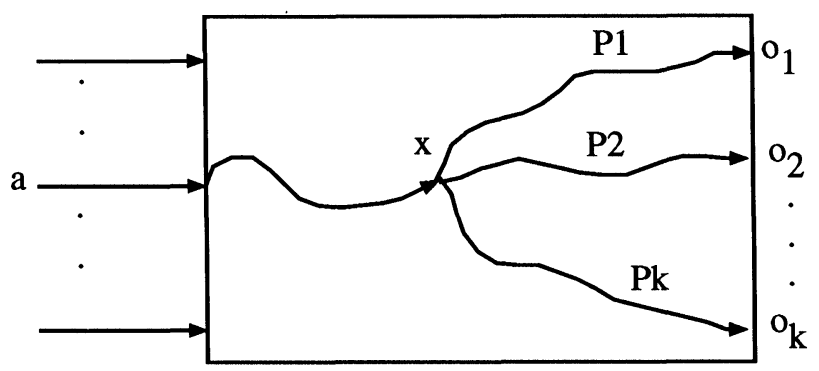

FIGURE 2. Logic Circuit Representation. 
$\operatorname{num} \_i n v(a, x)+\operatorname{num} \_i n v\left(x, o_{1}\right)=. .=$ num_inv $(a$, $\mathrm{x})+\operatorname{num} \_\operatorname{inv}\left(\mathrm{x}, \mathrm{o}_{\mathrm{k}}\right)$. This implies

$\operatorname{num} \_\operatorname{inv}\left(x, o_{1}\right)=\operatorname{num\_ inv}\left(x, o_{2}\right)=. .=\operatorname{num\_ inv}(x$, $\mathrm{o}_{\mathrm{k}}$ )

Therefore, there is same number of inversions between the fault site and the outputs which makes the fault produce unidirectional error. Q.E.D.

Corollary 1: If an input variable, $a$, is present in complemented or un-complemented form but not both in the output equations, then any fault located in a path leading from variable $a$ to the outputs will cause either single bit error or unidirectional multibit error.

Proof: Suppose that variable $a$ is presented in its uncomplemented form in all output equations, and suppose a fault $f$ occurred at node $x$. Since $x$ is affected by variable $a$, then by lemma 3 , num_inv(x, $\left.o_{i}\right)$ is the same for all outputs that are connected to $x$. Thus, this fault will cause unidirectional error.

\subsection{Output Encoding}

Another approach to prevent any two cubes from being $x$-bidirectional is output encoding. The idea behind output encoding is to ensure that the outputs corresponding to two input cubes $C_{i}$ and $C_{j}$ with $\operatorname{dist}\left(\mathrm{C}_{\mathrm{i}}, \mathrm{C}_{\mathrm{j}}\right)=1$, are not partially bidirectional.

To illustrate, let us consider Table $I$, where $Z_{1}$ and $\mathrm{Z}_{2}$ are the symbolic outputs. In this Table, $\mathrm{C}_{1}$ and $\mathrm{C}_{3}$ are $a$-bidirectional. Thus, by changing the output encoding for $Z_{1}$ from 100 to 110 , it can be guaranteed that the fault on line $a$ will not produce bidirectional error at the output.

Let us assume the specification of a logic circuit with $\mathrm{N}$ symbolic outputs. An algorithm for encoding the output so that there are no $m$-bidirectional input cubes is given below.

\section{Algorithm 3:}

1. Initialize $m$ to $[\lg N]$ where $N$ is the number of symbolic outputs.

2. Construct an undirected graph $G$ having the output symbols as vertices.

3. Connect Vertex $\mathrm{O}_{\mathrm{i}}$ to vertex $\mathrm{O}_{\mathrm{j}}$ if they are adjacent.

4. Construct a unidirectional graph $G_{m}$ with $2^{m}$ nodes.

5. If $\mathrm{Gm}$ does not cover $\mathrm{G}$, goto 6; else, an output encoding assignment is obtained from $\mathrm{G}_{\mathrm{m}}$. Exit.

6. Increment $\mathrm{m}$. Goto step 4.

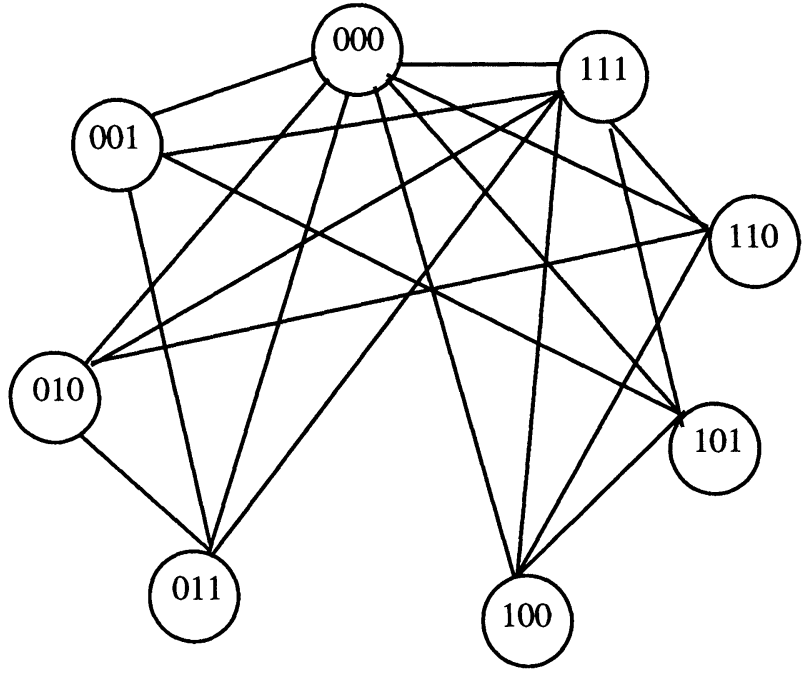

FIGURE 3. Unidirectional graph $\mathrm{G}_{3}$ for $\mathrm{m}=3$.

Figure 3 shows the unidirectional graph $\mathrm{G}_{3}$. Vertices which are represented by all 0 's or all 1's are connected to all other vertices because there is no other vertex which is partially bidirectional with these vertices. The number of edges for a vertex depends on the number of 1's in the representation of that vertex. If $\mathrm{k}$ is the number of 1's in an m-bit vertex, then the number of edges for that vertex is $\left(2^{k}-1\right)+\left(2^{(m-k)}-1\right)$

Table $\mathrm{V}$ shows that the number of edges each node has in a unidirectional graph as a function of $m$ and $\mathrm{k}$. For every $(\mathrm{m}, \mathrm{k})$ entry, the number of vertices is $\mathrm{m} ! /(\mathrm{k} ! \times(\mathrm{m}-\mathrm{k}) !)$. A fully connected subgraph of $\mathrm{G}_{\mathrm{m}}$ can have a maximum of $m+1$ nodes. For example, a fully connected subgraph of $\mathrm{G}_{3}$ can have a maximum of 4 nodes.

To illustrate the application of the above algorithm, let us consider the specification for 3-bit priority encoder shown in Table VI. Each vertex in Graph $\mathrm{G}$, Figure 4 , is connected to all other vertices. The unidirectional graph $\mathrm{G}_{2}$ does not cover $\mathrm{G}$. However,

TABLE V

Adjacency for Each Node as a Function of Space Dimension $(\mathrm{m})$ and number of 1 's $(\mathrm{k})$

\begin{tabular}{crrrrrrrrrr}
\hline & $\mathrm{k}=0$ & $\mathrm{k}=1$ & 2 & 3 & 4 & 5 & 6 & 7 & 8 & 9 \\
\hline $\mathrm{m}=1$ & 1 & 1 & & & & & & & & \\
2 & 3 & 2 & 3 & & & & & & & \\
3 & 7 & 4 & 4 & 7 & & & & & & \\
4 & 15 & 8 & 6 & 8 & 15 & & & & & \\
5 & 31 & 16 & 10 & 10 & 16 & 31 & & & & \\
6 & 63 & 32 & 18 & 14 & 18 & 32 & 63 & & & \\
7 & 127 & 64 & 34 & 22 & 22 & 34 & 64 & 127 & & \\
8 & 255 & 128 & 66 & 38 & 30 & 38 & 66 & 128 & 255 & \\
9 & 511 & 256 & 130 & 70 & 46 & 46 & 70 & 130 & 256 & 511 \\
\hline
\end{tabular}


TABLE VI

Specification of 3-bit Priority Encoder

\begin{tabular}{llccc}
\hline $\begin{array}{l}\text { Input } \\
\text { Cubes }\end{array}$ & $\mathrm{x}$ & $\mathrm{Inputs}$ & $\mathrm{z}$ & $\mathrm{s}$ \\
\hline C1 & 0 & 0 & 0 & O1 \\
C2 & 1 & - & - & O2 \\
C3 & 0 & 1 & - & O3 \\
C4 & 0 & 0 & 1 & O4 \\
\hline
\end{tabular}

$\mathrm{G}_{3}$ covers $\mathrm{G}$ (the graph in Figure 3 covers the graph in Figure 4); hence, three bits are needed for output encoding.

Since $G$ is fully connected, a fully connected subgraph containing 4 vertices of $\mathrm{G}_{3}$ is needed for output encoding. One possible choice is $(000,111$, 101, 100). Therefore, the outputs can be encoded as follows: $000 \rightarrow \mathrm{O}_{1}, 111 \rightarrow \mathrm{O}_{2}, 101 \rightarrow \mathrm{O}_{3}, 100 \rightarrow \mathrm{O}_{4}$.

Step 5 of Algorithm 3 is a graph embedding problem which is NP complete. Therefore solving this embedding problem by using the smallest $\mathrm{m}$ requires exhaustive search. A heuristic solution with polynomial complexity is proposed in this paper. This heuristic solution gives satisfactory results when applied to MCNC benchmark circuits. As the results show, in the worst case the number of bits used for encoding the output is only one bit more than the minimum number required. The nodes of graph $\mathrm{G}$ are the symbolic outputs which are denoted by $\mathrm{O}_{i}$ 's. The nodes of graph $G_{m}$ are $m$-bit vectors which are denoted by $V_{i}$ 's. The graph-embedding procedure is performed as follows:
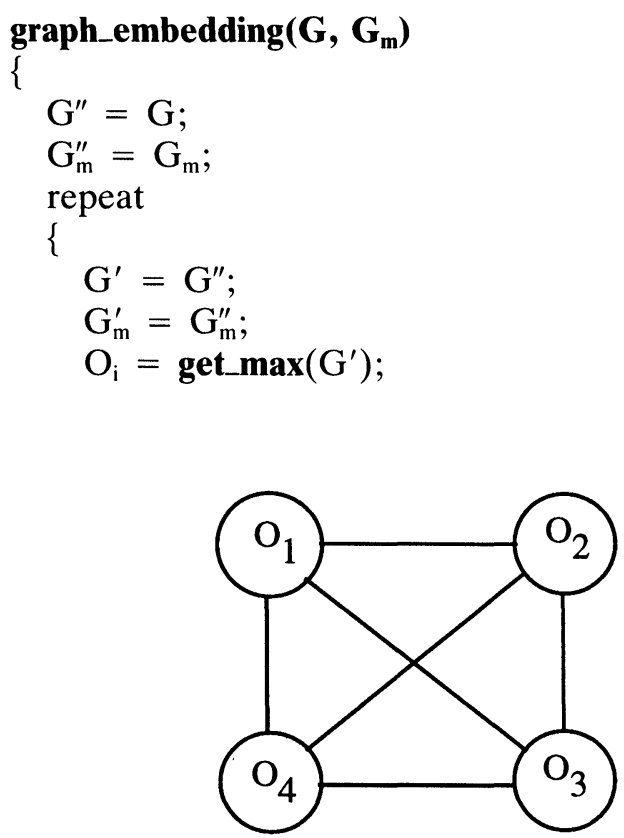

FIGURE 4. Graph G for 3-bit priority encoder. unidirectional_set $\left(\mathrm{O}_{\mathrm{i}}\right)=$ set of all nodes that are connected to $\mathrm{O}_{\mathrm{i}}$;

$\mathrm{V}_{\mathrm{i}}=$ get_max

unidirectional_set $\left(\mathrm{V}_{\mathrm{i}}\right)=$ set of all nodes that are connected to $\mathrm{V}_{\mathrm{i}}$;

if num_edges $\left(\mathrm{O}_{\mathrm{i}}\right) \leq$ num_edges $\left(\mathrm{V}_{\mathrm{i}}\right)$

\{

use the m-bit encoding for $\mathrm{V}_{\mathrm{i}}$ to encode output $\mathrm{O}_{\mathrm{i}}$;

form $G^{\prime \prime}$ which is a subgraph of $G^{\prime}$ with vertices as unidirectional_set $\left(\mathrm{O}_{\mathrm{i}}\right)$;

form $G_{m}^{\prime \prime}$ which is a subgraph of $G_{m}^{\prime}$ with vertices as unidirectional_set $\left(\mathrm{V}_{\mathrm{i}}\right)$;

graph_embedding $\left(G^{\prime \prime}, G_{\mathbf{m}}^{\prime \prime}\right)$;

$\mathrm{G}^{\prime \prime}=\mathrm{G}^{\prime}$ - (node $\mathrm{O}_{\mathrm{i}}$ and unidirectional_set $\left(\mathrm{O}_{\mathrm{i}}\right)$ and all edges joining the edges of these nodes);

$\mathrm{G}_{\mathrm{m}}^{\prime \prime}=\mathrm{G}_{\mathrm{m}}^{\prime}$ - (node $\mathrm{V}_{\mathrm{i}}$ and unidirectional_ $\operatorname{set}\left(V_{i}\right)$ and all edges joining the edges of these

\} nodes)

else

\{

$\mathrm{m}=\mathrm{m}+1$

undecode all symbolic outputs;

$\mathrm{G}^{\prime \prime}=\mathrm{G}$;

$\mathrm{G}_{\mathrm{m}}^{\prime \prime}=\mathrm{G}_{\mathrm{m}}$;

\} until all outputs are encoded; \} .

The routine get_max( ) returns the node that has the largest number of edges. The routine num edges( ) returns the number of edges for the node. Unidirectional_set $\left(\mathrm{O}_{\mathrm{i}}\right)$ is the set of nodes that are joined to $\mathrm{O}_{i}$ by an edge. The dimension of $\mathrm{G}_{\mathrm{m}}$ will increase by 1 when the if condition in graph_embedding ( ) fails; in other words, when node $\mathrm{O}_{i}$ in $\mathrm{G}^{\prime}$ could not be mapped to node $V_{i}$ in $G_{m}^{\prime}$ because $O_{i}$ has more edges than $\mathrm{V}_{\mathrm{i}}$. When the if condition is true in the procedure, graph_embedding( ) will be recursively called for embedding smaller sizes graphs because the cardinality of the unidirectional_set $\left(\mathrm{O}_{\mathrm{i}}\right)$ is always less than the number of nodes in the graph containing $\mathrm{O}_{\mathrm{i}}$.

Lemma 4: The complexity of Algorithm 3 does not exceed the order of $\mathrm{N}^{4}$ where $\mathrm{N}$ is the number of symbolic outputs.

Proof: The number of edges/nodes in graph $\mathrm{G}$ are used in the estimation of the complexity because the number of edges/nodes in $G_{m}$ are equal to a constant time the number of edges/nodes in G. The complexity of constructing graph $\mathrm{G}$ in Algorithm 3 is of the order of $\mathrm{N}^{2}$ because for each node in $\mathrm{G}, \mathrm{N}-1$ 
checks are made to see if that node is connected to the other nodes (Step 3 in the algorithm). Similarly, the construction of $G_{m}$ has polynomial complexity. Each call to the graph_embedding( ) procedure is linearly related to the number of nodes plus the number of edges because routine get_max( ) is linearly related to the number of nodes $(\mathrm{N})$, and routine num _edges( ) takes constant time. The unidirectional_set ( ) is constructed in a time linearly related to the number of edges $(|\mathrm{E}|)$. It should be noted here that the complexity of the above procedures depends on how the graphs have been stored. When the if condition is always true in the procedure, graph_embedding( ) will be recursively called for a maximum of $\mathrm{N}-1$ times with each call requiring steps of order $\mathrm{N}$. Thus, the complexity is of the order of $\mathrm{N}(\mathrm{N}+|\mathrm{E}|)$. The worst case occurs when $G$ is fully connected; thus $|E|=N^{2} / 2$ and the complexity of step 5 will be of the order of $\mathrm{N}^{3}$. When the if condition fails in the last recursive call for graph_embedding( ), order of $\mathrm{N}^{3}$ operations are wasted and $\mathrm{m}$ is increased by 1 and the whole procedure is repeated. The if condition will be true when $m$ is equal to $N-1$ because a fully connected subgraph from $G_{m}$ of $N$ nodes can be found. Consequently $G$ will be covered by $G_{m}$. Therefore, the if condition can fail up to $(\mathrm{N}-1-$ $[\lg N])$ times, and for each iteration, operations of the order of $\mathrm{N}^{3}$ are needed, thus the worst case complexity is order of $\mathrm{N}^{4}$. Q.E.D.

Let us consider the application of the graph embedding procedure to the 3-bit priority encoder shown in Table VI. The graph $\mathrm{G}$ for the 3-bit priority encoder is shown in Figure 4. The procedure will try first graph_embedding $\left(G, G_{2}\right)$. In the first iteration, 00 will be used for $\mathrm{O}_{1}$ encoding. Thus, unidirectional $\operatorname{set}\left(\mathrm{O}_{1}\right)=\left\{\mathrm{O}_{2}, \mathrm{O}_{3}, \mathrm{O}_{4}\right\}$, and unidirectional_set $(00)$ $=\{11,01,10\}$. Next, 11 will be used for $\mathrm{O}_{2}$ encoding. Thus, unidirectional_set $\left(\mathrm{O}_{2}\right)=\left\{\mathrm{O}_{3}, \mathrm{O}_{4}\right\}$, and unidirectional_set $(11)=\{01,10\}$. In this case, $\mathrm{O}_{3}$ could not be mapped to either 01 or 10 because num _edges $\left(\mathrm{O}_{3}\right)(=1)$ is greater than num_edges $(01)(=$ 0 ). Thus, the dimension is increased from 2 to 3 , and graph_embedding $\left(G, G_{3}\right)$ is called. It could be easily seen how $000 \rightarrow \mathrm{O}_{1}, 111 \rightarrow \mathrm{O}_{2}, 101 \rightarrow \mathrm{O}_{3}, 100 \rightarrow$ $\mathrm{O}_{4}$.

Lemma 5: If a prime and irredundant combinational logic circuit has its outputs encoded using algorithm 2, then any stuck-at fault (internal or at the input lines) will result in either single bit error or unidirectional multibit error at the output regardless of the way the circuit is implemented.
Proof: Algorithm 2 guarantees the elimination of $m$-bidirectional input cubes; therefore, by lemma 2 , all single faults at the input lines will cause unidirectional error at the output. On the other hand, since the circuit is prime and irredundant, it has to be $100 \%$ testable. Therefore, for each node, there exists at least one input pattern that makes a sensitizable path from an input(s) through that node to an output(s). If there is only one sensitizable path for any input pattern from an input passing through node $x$ to an output (there might be another path, but it cannot be sensitizable for the same input pattern), a fault at node $x$ will produce single bit error since the fault only propagates to one output. In addition, if there is $\mathrm{k}$ sensitizable paths from input $a$ through node $x$ to $\mathrm{k}$ different outputs (Figure 2), an event at input $a$ will cause an event at node $x$ which will also cause events at the $\mathrm{k}$ outputs. An event at input $a$ cannot cause a bidirectional change at the output due to the output assignment in algorithm 2. Thus, a transition or a stuck-at fault at node $x$ cannot cause bidirectional error under the given input. Moreover, there might be other paths from an input different from $a$ through node $x$ and to some outputs; however, similar arguments can be made to prove that a fault at node $x$ cannot cause bidirectional error. Consequently, all possible single stuck-at fault will either cause single bit error or unidirectional multibit error at the output. Q.E.D.

The following lemma summarizes the main features of the proposed techniques.

Lemma 6: If a combinational circuit is designed such that all faults at the inputs which create bidirectional error at the output are removed, any fault in the circuit, internal or at the inputs, will result in either single bit error or unidirectional multibit error at the output irrespective of the way the circuit is implemented.

Proof: The proof of this lemma is similar to the proof of lemma 5.

A detailed example is considered below to illustrate the application of the proposed techniques.

Example: In this example the application of the encoding techniques on rd53 MCNC benchmark circuit will be considered. Table VIIa shows the description of the benchmark circuit. rd53 circuit has 5 inputs, 3 outputs, 32 product terms, 32 different input patterns and 6 different output patterns. An 
TABLE VII

PLA Descriptions of the Same Circuit Using Different Input and Output Encoding Schemes

(a) Benchmark description

\begin{tabular}{|ll|}
\hline .1 5 & \\
\hline .03 & \\
\hline .p 32 & \\
\hline $1-111$ & $1 \sim \sim$ \\
\hline $11-11$ & $1 \sim \sim$ \\
\hline $1111-$ & $1 \sim \sim$ \\
\hline $111-1$ & $1 \sim \sim$ \\
\hline-1111 & $1 \sim \sim$ \\
\hline $01-01$ & $\sim \sim 1$ \\
\hline-0110 & $\sim \sim 1$ \\
\hline $001-1$ & $\sim \sim 1$ \\
\hline $1-001$ & $\sim \sim 1$ \\
\hline $1-100$ & $\sim \sim 1$ \\
\hline $110-0$ & $\sim \sim 1$ \\
\hline $011-0$ & $\sim \sim 1$ \\
\hline $1001-$ & $\sim \sim 1$ \\
\hline $0-011$ & $\sim \sim 1$ \\
\hline-1010 & $\sim \sim 1$ \\
\hline-0101 & $\sim \sim 1$ \\
\hline 01110 & $\sim 1 \sim$ \\
\hline 00010 & $\sim 1 \sim$ \\
\hline 01000 & $\sim 1 \sim$ \\
\hline 11111 & $\sim 1 \sim$ \\
\hline 00100 & $\sim 1 \sim$ \\
\hline 00111 & $\sim 1 \sim$ \\
\hline 11100 & $\sim 1 \sim$ \\
\hline 11010 & $\sim 1 \sim$ \\
\hline 01101 & $\sim 1 \sim$ \\
\hline 01011 & $\sim 1 \sim$ \\
\hline 10110 & $\sim 1 \sim$ \\
\hline 10000 & $\sim 1 \sim$ \\
\hline 11001 & $\sim 1 \sim$ \\
\hline 00001 & $\sim 1 \sim$ \\
\hline 10101 & $\sim 1 \sim$ \\
\hline 10011 & $\sim 1 \sim$ \\
\hline
\end{tabular}

(b) Input encoding.

\begin{tabular}{|c|c|}
\hline .17 & \\
\hline .03 & \\
\hline p 32 & \\
\hline 111-..- & $1 \sim \sim$ \\
\hline 11-1-.. & $1 \sim \sim$ \\
\hline 11--1-- & $1 \sim \sim$ \\
\hline $11---1-$ & $1 \sim \sim$ \\
\hline $11 \ldots 1$ & $1 \sim \sim$ \\
\hline $1-11-$. & $\sim \sim 1$ \\
\hline 1-1-1-- & $\sim \sim 1$ \\
\hline 1-1--1- & $\sim \sim 1$ \\
\hline $1-1---1$ & $\sim \sim 1$ \\
\hline $1--11--$ & $\sim \sim 1$ \\
\hline $1--1-1-$ & $\sim \sim 1$ \\
\hline $1--1--1$ & $\sim \sim 1$ \\
\hline $1--11-$ & $\sim \sim 1$ \\
\hline $1--1-1$ & $\sim \sim 1$ \\
\hline $1-\cdots 11$ & $\sim \sim 1$ \\
\hline$-111-$. & $\sim \sim 1$ \\
\hline$-11-1-$ & $\sim 1 \sim$ \\
\hline$-11--1-$ & $\sim 1 \sim$ \\
\hline$-11---1$ & $\sim 1 \sim$ \\
\hline$-1-11-$ & $\sim 1 \sim$ \\
\hline$-1-1-1-$ & $\sim 1 \sim$ \\
\hline$-1-1--1$ & $\sim 1 \sim$ \\
\hline$-1--11-$ & $\sim 1 \sim$ \\
\hline$-1--1-1$ & $\sim 1 \sim$ \\
\hline$-1--11$ & $\sim 1 \sim$ \\
\hline$--111-$ & $\sim 1 \sim$ \\
\hline$--11-1-$ & $\sim 1 \sim$ \\
\hline$--11--1$ & $\sim 1 \sim$ \\
\hline$--1-11-$ & $\sim 1 \sim$ \\
\hline$--1-1-1$ & $\sim 1 \sim$ \\
\hline$--1--11$ & $\sim 1 \sim$ \\
\hline$---111-$ & $\sim 1 \sim$ \\
\hline
\end{tabular}

(c) Symbolic outputs

\begin{tabular}{|ll|}
\hline 00000 & O0 \\
\hline 00001 & O1 \\
\hline 00010 & O1 \\
\hline 00011 & O2 \\
\hline 00100 & O1 \\
\hline 00101 & O2 \\
\hline 00110 & O2 \\
\hline 00111 & O3 \\
\hline 01000 & O1 \\
\hline 01001 & O2 \\
\hline 01010 & O2 \\
\hline 01011 & O3 \\
\hline 01100 & O2 \\
\hline 01101 & O3 \\
\hline 01110 & O3 \\
\hline 01111 & O4 \\
\hline 10000 & O1 \\
\hline 10001 & O2 \\
\hline 10010 & O2 \\
\hline 10011 & O3 \\
\hline 10100 & O2 \\
\hline 10101 & O3 \\
\hline 10110 & O3 \\
\hline 10111 & O4 \\
\hline 11000 & O2 \\
\hline 11001 & O3 \\
\hline 11010 & O3 \\
\hline 11011 & O4 \\
\hline 11100 & O3 \\
\hline 11101 & O4 \\
\hline 11110 & O4 \\
\hline 11111 & O5 \\
\hline
\end{tabular}

(d) Output encoding.

\begin{tabular}{|c|c|}
\hline .15 & \\
\hline .03 & \\
\hline. $\mathrm{p} 32$ & \\
\hline 00000 & 111 \\
\hline 00001 & 110 \\
\hline 00010 & 110 \\
\hline 00011 & 010 \\
\hline 00100 & 110 \\
\hline 00101 & 010 \\
\hline 00110 & 010 \\
\hline 00111 & 011 \\
\hline 01000 & 110 \\
\hline 01001 & 010 \\
\hline 01010 & 010 \\
\hline 01011 & 011 \\
\hline 01100 & 010 \\
\hline 01101 & 011 \\
\hline 01110 & 011 \\
\hline 01111 & 001 \\
\hline 10000 & 110 \\
\hline 10001 & 010 \\
\hline 10010 & 010 \\
\hline 10011 & 011 \\
\hline 10100 & 010 \\
\hline 10101 & 011 \\
\hline 10110 & 011 \\
\hline 10111 & 001 \\
\hline 11000 & 010 \\
\hline 11001 & 011 \\
\hline 11010 & 011 \\
\hline 11011 & 001 \\
\hline 11100 & 011 \\
\hline 11101 & 001 \\
\hline 11110 & 001 \\
\hline 11111 & 000 \\
\hline
\end{tabular}

unconstrained boolean minimization using MIS [10] is applied first to the original description of the circuit as described in the benchmark, which produces the following results:

lits(sop) $=45$ lits(fac) $=36$, where lits(sop) is the number of literals in sum-of-product form, and lits $(\mathrm{fac})$ is the number of literals in the factored form.
The output equations are:

$$
\begin{aligned}
\{\mathrm{v} 5.0\}= & (5) \mathrm{v} 0 \mathrm{v} 1+\mathrm{v} 0 \mathrm{v} 2 \mathrm{v} 3 \mathrm{v} 4+\mathrm{v} 1 \mathrm{v} 2 \mathrm{v} 3 \mathrm{v} 4 \\
\{\mathrm{v} 5.1\}= & (11) \mathrm{v} 0+(11)^{\prime} \mathrm{v} 0{ }^{\prime} \\
\{\mathrm{v} 5.2\}= & \{\mathrm{v} 5.0\}^{\prime}\{\mathrm{v} 5.1\}^{\prime} \mathrm{v} 1+\{\mathrm{v} 5.0\}^{\prime}(5)+\{\mathrm{v} 5.0\}^{\prime} \mathrm{v} 0 \\
& \mathrm{v} 2+\{\mathrm{v} 5.0\}^{\prime} \mathrm{v} 0 \mathrm{v} 3+\{\mathrm{v} 5.0\}^{\prime} \mathrm{v} 0 \mathrm{v} 4
\end{aligned}
$$




$$
\begin{aligned}
(4) & =(14) \mathrm{v} 1+(14)^{\prime} \mathrm{v} 1^{\prime} \\
(5) & =(14) \mathrm{v} 4+(14)^{\prime} \mathrm{v} 2 \\
(11) & =(4) \mathrm{v} 4^{\prime}+(4)^{\prime} \mathrm{v} 4 \\
(14) & =\mathrm{v} 2 \mathrm{v} 3^{\prime}+\mathrm{v} 2^{\prime} \mathrm{v} 3
\end{aligned}
$$

$\mathrm{v} 0, \mathrm{v} 1, \mathrm{v} 2$, v3, and v4 are the inputs; $\{\mathrm{v} 5.0\},\{\mathrm{v} 5.1\}$ and $\{v 5.2\}$ are the outputs; (4), (5), (11) and (14) are internal nodes. Each node is written in a sum-ofproduct form in terms of the input variables and the other nodes. In this realization, there exist some faults that create bidirectional error at the outputs. If, for instance, the input pattern is v0v1v2v3v4 = 11001, the internal nodes and the outputs will be as follows: $(14)=(4)=(5)=0 ;(11)=1 ;\{v 5.0\}=$ $0 ;\{\mathrm{v} 5.1\}=\{\mathrm{v} 5.2\}=1$. If, however, input $\mathrm{v} 2$ is s-a1 , the value of the nodes will be changed to: $(14)=$ (4) $=(5)=0 ;(11)=0 ;(11)=0 ;\{\mathrm{v} 5.0\}=1 ;\{\mathrm{v} 5.1\}$ $=\{v 5.2\}=0$. Thus, in the presence of the fault, the outputs change from 011 to 100 . Similarly, a stuckat-1 at node (14) will create bidirectional error when 11001 is applied at the input.

The standard cell representation of the circuit results in an area of 110400 Lambdas $(230 \times 480$ Lambdas).

As mentioned previously, m-out-of- $n$ codes are used for input encoding. To minimize the number of input lines, we choose $m$ to be $[n / 2]$. Since the number of input combinations is 32 , 3-out-of-7 code is used for encoding the inputs as shown in Table VIIb. It can be easily verified that by using this input encoding, either single bit error or unidirectional multibit error at the output will result in presence of a stuck-at fault. An unconstrained boolean minimization using MIS is applied, and the following results are obtained:

$$
\operatorname{lits}(\mathrm{sop})=41 \operatorname{lits}(\mathrm{fac})=32
$$

The output equations are:

$$
\begin{aligned}
\{\mathrm{v} 7.0\}= & (20) \mathrm{v} 0 \mathrm{v} 1+\mathrm{v} 0 \mathrm{v} 1 \mathrm{v} 2+\mathrm{v} 0 \mathrm{v} 1 \mathrm{v} 3 \\
\{\mathrm{v} 7.1\}= & (19) \mathrm{v} 1+(19) \mathrm{v} 2+(20) \mathrm{v} 1 \mathrm{v} 2 \\
& +\mathrm{v} 3 \mathrm{v} 4 \mathrm{v} 5 \\
\{\mathrm{v} 7.2\}= & (19) \mathrm{v} 0+(20) \mathrm{v} 0 \mathrm{v} 2+\mathrm{v} 0 \mathrm{v} 2 \mathrm{v} 3 \\
& +\mathrm{v} 1 \mathrm{v} 2 \mathrm{v} 3
\end{aligned}
$$

$$
\begin{aligned}
& (19)=(20) \mathrm{v} 3+\mathrm{v} 4 \mathrm{v} 5+\mathrm{v} 4 \mathrm{v} 6+\mathrm{v} 5 \mathrm{v} 6 \\
& (20)=\mathrm{v} 4+\mathrm{v} 5+\mathrm{v} 6
\end{aligned}
$$

In these output equations, no variable appear in both complemented and uncomplemented form. Thus, these equations are monotonic which guarantees that every fault will create either single bit error or unidirectional multibit error at the output (Lemma 3).

The standard cell layout for this realization results in an area of 109440 Lambdas $(235 \times 384$ Lambdas $)$. Thus, the overhead is $(109440-110400) / 110400=$ $-0.87 \%$; i.e., the area is actually reduced.

Table VIIc shows the description of $\mathbf{r d 5 3}$ when the inputs cubes are expanded and a symbolic output is assigned to each output pattern. The graph constructed from Algorithm 3 is shown in Figure 5.

Since $\mathrm{G}_{3}$ covers $\mathrm{G}$, a possible output encoding is as follows:

$$
\begin{array}{lll}
111 \longrightarrow \mathrm{O} 0 & 110 \longrightarrow \mathrm{O} 1 & 010 \longrightarrow \mathrm{O} 2 \\
011 \longrightarrow \mathrm{O} 3 & 001 \longrightarrow \mathrm{O} 4 & 000 \longrightarrow \mathrm{O} 5
\end{array}
$$

We applied an unconstrained boolean minimization using MIS, and the following results are obtained:

$$
\operatorname{lits}(\mathrm{sop})=40 \operatorname{lits}(\mathrm{fac})=36
$$

The output equations are:

$$
\begin{aligned}
& \{\mathrm{v} 5.0\}=(32)(39)+(33)(38)^{\prime} \mathrm{v} 4^{\prime} \\
& \{\mathrm{v} 5.1\}=(32)+(33)(37)+(33) \mathrm{v} 4^{\prime}+(39)
\end{aligned}
$$

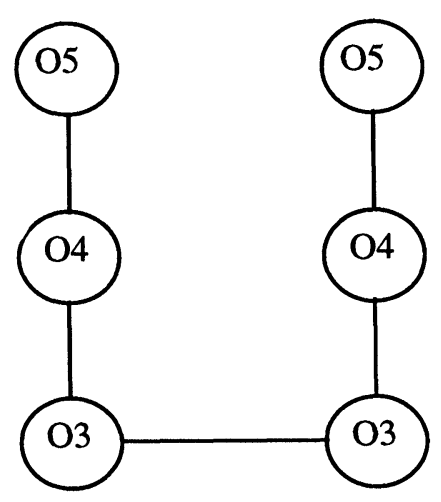

FIGURE 5. Graph G for the circuit in Table VIIc. 


$$
\begin{aligned}
\{\mathrm{v} 5.2\}= & (32)^{\prime}(33)(39)^{\prime}+(33)^{\prime} \mathrm{v} 2 \mathrm{v} 4^{\prime} \\
& +(33)^{\prime} \mathrm{v} 2^{\prime} \mathrm{v} 3+(33)^{\prime} \mathrm{v} 3^{\prime} \mathrm{v} 4 \\
& +(37)^{\prime}(39) \mathrm{v} 4+(38)^{\prime}(39) \mathrm{v} 4^{\prime} \\
(32)= & (37) \mathrm{v} 4^{\prime}+(38)^{\prime} \\
(33)= & \mathrm{v} 0^{\prime}+\mathrm{v} 1^{\prime} \\
(37)= & \mathrm{v} 2^{\prime}+\mathrm{v} 3^{\prime} \\
(38)= & \mathrm{v} 2+\mathrm{v} 3 \\
(39)= & \mathrm{v} 0^{\prime} \mathrm{v} 1^{\prime}
\end{aligned}
$$

Node (33) is used in an uncomplemented form in the equations for $\{\mathrm{v} 5.0\},\{\mathrm{v} 5.1\}$, and $\{\mathrm{v} 5.2\}$; however, its is used in complemented form in $\{v 5.2\}$. In this case, the num_inv $((33),\{\mathrm{v} 5.0\})=0$ whereas num $\operatorname{inv}((33),\{v 5.2\})=1$. We will prove that if one of the paths from node (33) to an output where num inv ((33), output $\}=0$ is sensitizable, all other paths from (33)' to the outputs are not sensitizable. If the path from (33) to $\{\mathrm{v} 5.1\}$ is sensitizable, then $\mathrm{v} 4$ should be 0 or (37) should be 1 , and both (39) and (32) should be 0 . When node (39) $=0$, then

$$
\begin{aligned}
\{\mathrm{v} 5.2\}= & (33)+(33)^{\prime} \mathrm{v} 2 \mathrm{v} 4^{\prime}+(33)^{\prime} \mathrm{v} 2^{\prime} \mathrm{v} 3 \\
& +(33)^{\prime} \mathrm{v} 3^{\prime} \mathrm{v} 4 \\
= & (33)+\mathrm{v} 2 \mathrm{v}^{\prime}+\mathrm{v} 2^{\prime} \mathrm{v} 3+\mathrm{v} 3^{\prime} \mathrm{v} 4 .
\end{aligned}
$$

(33) is only used in an uncomplemented form, and the path from (33)' to $\{v 5.2\}$ is masked. Therefore, there will be the same number of inversions between node (33) and the outputs.

Similarly, it can be shown that if one of the paths from a node to an output, where the number of inversions is even(odd) is sensitizable, then all other paths from that node to the outputs where number of inversions is odd (even) are not sensitizable.

The standard cell layout for the above realization results in an area of 90688 Lambdas $(218 \times 416$ Lambdas). Thus the area overhead is (90688 $110400) / 110400=-18 \%$.

Note that the area overhead for the standard cell layout is not the same as the overhead for lits(fac) (Tables IX and X). This is because the area in the number of literals does not take into account the routing needed in the layout. However, lits(fac) still give a good measure of the overhead of different realization.

\section{RESULTS}

The number of literals in the factored form (multilevel realization) is approximately twice the number of transistors used in CMOS implementation because each literal will be an input to two transistors (one $\mathrm{n}$-type and the other is p-type). Therefore, the results reported here are the number of literals in both factored form and sum-of-product form. As far as we are aware, no other input/output encoding schemes are available that have the same objectives as that proposed in this paper. Thus, for each benchmark circuit, we will have three realizations: 1 ) direct implementation of the circuit as described in the benchmark, 2) implementation of the circuit after input encoding and 3) implementation of circuit after applying Algorithm 3. The results will be reported as the number of literals for each realization. The results of realization 2) and 3) are compared to the results of realization 1). The statistics of the benchmarks are given in Table VIII where \#inp is the number of input lines, \#out is the number of output lines, \#pro is the number of product terms, \#diff_i is the number of different inputs present in the pla description, and \#diff_o is the number of different outputs after expanding the input cubes. An unconstrained boolean minimization has been applied to the different circuits by using the multilevel logic synthesis tool MIS [10].

Table IX shows the number of literals obtained for realizations 1) and 2). \#lit(sop) is the number of literals in sum of product form, \#lit(fac) is the number of products in the factored form.

The percentage overhead is given for literal counts obtained from both sum-of-product and factored representation. Negative overhead indicates that the encoded circuit has fewer literals than the original circuit. It is clear from the experimental results that one can apply the input encoding to ensure every fault result in either a single bit error or unidirectional multibit error at the output, without increasing and

TABLE VIII

Statistics of Benchmark Examples

\begin{tabular}{lrrrrr}
\hline Example & \#inp & \#out & \#pro & \#diff_i & \#diff_o \\
\hline 5xp1 & 7 & 10 & 75 & 70 & 128 \\
apex1 & 45 & 45 & 206 & 205 & \\
bw & 5 & 28 & 87 & 76 & \\
clip & 9 & 5 & 167 & 166 & \\
con1 & 7 & 2 & 9 & 9 & 4 \\
duke2 & 22 & 29 & 87 & 87 & \\
misex1 & 8 & 7 & 32 & 18 & \\
misex2 & 25 & 18 & 29 & 29 & \\
rd53 & 5 & 3 & 32 & 32 & 6 \\
rd73 & 7 & 3 & 141 & 141 & 8 \\
table3 & 14 & 14 & 175 & 175 & \\
\hline
\end{tabular}


TABLE IX

Literal Count for Original and Input Encoded Circuits Plus the Overhead

\begin{tabular}{|c|c|c|c|c|c|c|c|}
\hline \multirow[b]{2}{*}{ Example } & \multicolumn{2}{|c|}{$\begin{array}{c}\begin{array}{c}\text { Original } \\
\text { description }\end{array} \\
\end{array}$} & \multicolumn{3}{|c|}{$\begin{array}{c}\text { Input } \\
\text { Encoding } \\
\text { (m-out-of-n) }\end{array}$} & \multicolumn{2}{|c|}{$\operatorname{ovd}(\%)$} \\
\hline & $\begin{array}{c}\text { \#lit } \\
\text { (sop) }\end{array}$ & $\begin{array}{c}\text { \#lit } \\
(\mathrm{fac})\end{array}$ & $\begin{array}{c}\text { \#lit } \\
\text { (sop) }\end{array}$ & $\begin{array}{c}\text { \#lit } \\
\text { (fac) }\end{array}$ & \#inp & sop & fac \\
\hline $5 x p 1$ & 165 & 123 & 148 & 119 & 8 & -10.3 & -3.2 \\
\hline apex1 & 1822 & 1557 & 1315 & 1081 & 10 & -27.8 & -30.6 \\
\hline bw & 173 & 160 & 228 & 200 & 9 & 31.7 & 25 \\
\hline clip & 190 & 141 & 125 & 97 & 10 & -34.2 & -31.2 \\
\hline con1 & 23 & 19 & 13 & 11 & 5 & -43.5 & -42.1 \\
\hline duke2 & 532 & 433 & 440 & 379 & 9 & -17.3 & -12.5 \\
\hline $\operatorname{misex} 2$ & 113 & 103 & 74 & 66 & 7 & -34.5 & -35.9 \\
\hline rd53 & 45 & 36 & 41 & 32 & 7 & -8.9 & -11.1 \\
\hline $\mathrm{rd} 73$ & 108 & 80 & 111 & 82 & 10 & 2.78 & 2.5 \\
\hline table3 & 1019 & 838 & 740 & 567 & 10 & -27.4 & -32.3 \\
\hline total & 4190 & 3490 & 3235 & 2634 & & -19.4 & -24.5 \\
\hline
\end{tabular}

in most cases decreasing the overhead. The output encoding algorithm was applied to a few benchmark circuits to get some estimate of the overhead. Table $\mathrm{X}$ shows the results of these benchmarks.

In Table X, \#out is the number of output encoding bits used in the algorithm, ovd is the percentage overhead. As in input encoding, the average overhead for the benchmarks circuits is negative, which indicates that on average output encoded circuits require less overhead than the original circuit description. In addition, the number of bits used for encoding the output is only one bit more than the minimum number of bits required. Finally, the output encoding algorithm gives better results than the input encoding algorithm because the output algorithm assign unidirectional codes for two adjacent outputs; this assignment will make one output dominant resulting in fewer number of literals in the final circuit [11].

\section{CONCLUSION}

Input and output encoding techniques proposed in this paper guarantee that all stuck-at faults, internal or at the inputs, will cause either single bit error or

TABLE X

Literal Count and Overhead for Output Encoding Algorithm

\begin{tabular}{rcrrrr}
\hline Ex. & \#out & $\begin{array}{c}\text { \#lit } \\
\text { (sop) }\end{array}$ & $\begin{array}{c}\text { \#lit } \\
\text { (fac) }\end{array}$ & $\begin{array}{c}\text { ovd } \\
\text { (sop) }\end{array}$ & \multicolumn{1}{c}{$\begin{array}{c}\text { ovd } \\
\text { (fac) }\end{array}$} \\
\hline 5xp1 & 8 & 24 & 24 & $-84.6 \%$ & $-80.5 \%$ \\
con1 & 3 & 31 & 25 & $34.7 \%$ & $31.6 \%$ \\
rd53 & 3 & 40 & 36 & $-11.1 \%$ & $0.0 \%$ \\
rd73 & 3 & 78 & 73 & $-27.8 \%$ & $-8.7 \%$ \\
total & & 173 & 158 & $-49.2 \%$ & $-38.8 \%$ \\
\hline
\end{tabular}

unidirectional multibit error at the output. Previously published work in this area was restricted to AND/OR type circuits, and both inputs and their inversions were assumed fault-free. Thus, there are no schemes or algorithms for output or input encoding that have the same objectives as presented in this paper. The techniques proposed allow multilevel implementation as well as consider the possibility of faults at the input lines. The input encoding algorithm can be applied to arbitrary circuits because mout-of-n code is used for input encoding, so the increase in the input lines is not significant. The worst case theoretical complexity of Algorithm 3 is $\mathrm{N}^{4}$; however, the average experimental complexity in $\mathrm{N}^{2}$ (the number of bits used for encoding the output is one bit more than the minimum number of bits required). Therefore, Algorithm 3 can be efficiently applied to a circuit description that has up to few hundreds symbolic outputs.

\section{References}

[1] Y. Savaria, N.C. Rumin, J. Hayes, and V. Agrawal, "Softerror filtering: A solution to the reliability problem of future VLSI logic circuits," IEEE Proc., vol. 74, no. 5, pp. 669683, May 1986.

[2] M. Yen, W. Fuchs, and J. Abraham, "Designing for concurrent error detection in VLSI: Application to a microprogram control unit," IEEE Journal of Solid-State Circuits, vol. SC-22, pp. 595-605, August 1987.

[3] J. Smith and G. Metze, "The design of totally self-checking combinational circuits," in Proc. Int. Symp. Fault-Tolerant Computing, Los Angeles, CA, pp. 130-134, June 1977.

[4] M. Diaz, "Design of totally self-checking and fail safe sequential machines," in Proc. Int. Symp. Fault-Tolerant Computing, Urbana, IL, pp. 9-24, June 1974.

[5] J. Smith and P. Lam, "A theory of totally self-checking system design," IEEE Trans. on Computers, vol. C-32, pp. 831-844, September 1983.

[6] C. Frieman, "Optimal error detecting codes for completely asymmetric binary channels," Information and Control, vol. 5, pp. 64-71, March 1962.

[7] H. Dong, "Modified Berger codes for detection of unidirectional error," in Proc. Int. Symp. Fault-Tolerant Comput., Santa Clara, CA, pp. 317-320, June 1982.

[8] S. Devadas, H.T. Ma, A.R. Newton, and A.L. Sangiovanni-Vincentelli, "Irredundant sequential machines via optimal logic synthesis," IEEE Trans. Computer-Aided Design, vol. 9, pp. 8-17, Jan. 1990.

[9] S. Devadas, and K. Keutzer, "A unified approach to the synthesis of fully testable sequential machines," IEEE Trans. Computer-Aided Design, vol. 10, pp. 39-50, Jan. 1991.

[10] R.K. Brayton, R. Rudell, A. Sangiovanni-Vincentelli, and A.R. Wang, "MIS: A multiple-level logic optimization program," IEEE Trans. on Computer-Aided Design, vol. 7, pp. 1062-1081, Nov. 1987.

[11] S. Devadas, and A.R. Newton, "Exact Algorithms for output encoding, state assignment and four-level Boolean minimization," IEEE Trans. Computer-Aided Design, vol. 10, pp. 13-27, Jan. 1991.

[12] K. Keutzer, S. Malik, and A. Saldanha, "Is redundancy necessary to reduce delays?," IEEE Trans. Computer Aided-Design, vol. 10, pp. 427-435, April 1991. 
[13] K. Bartlett, R.K. Brayton, G.D. Hachtel, R.M. Jacoby, C.R. Morrison, R.L. Rudell, A. Sangiovanni-Vincentelli, and A.R. Wang, "Multi-level logic minimization using implicit don't cares," IEEE Trans. Computer-Aided Design, vol. 7, pp. 723-740, June 1988.

[14] J.E. Smith and G. Metze, "Strongly fault secure logic networks," IEEE Trans. on Computers, vol. C27, pp. 491499, June 1987.

[15] S. Pagey, S.D. Sherlekar and G. Venkatesh, "A Methodology for the design of SFS/SCD circuits for a class of unordered codes," Journal of Electronic Testing: Theory and Application, 2, pp. 261-277, 1991.

\section{Biographies}

FADI BUSABA is an assistant professor in the department of Electrical Engineering at North Carolina A\&T State University.
His research interests include fault-tolerant computing, selfchecking and synthesis for testability. He holds a BE from American University of Beirut, an M.S.E.E. from North Carolina Agricultural and Technical State University, and a Ph.D. in Computer Engineering from North Carolina State University at Raleigh. He received MCNC Fellowship and an IEEE member.

PARAG K. LALA is a professor in the department of Electrical Engineering at North Carolina Agricultural and Technical State University. His research interests include test generation/testability, fault-tolerant computing, digital system design, and selfchecking design. He authored Fault-Tolerant and Fault-Testable Hardware Design and Digital System Design Using PLDs, both published by Prentice-Hall, Inc. He holds an MSc in Electrical Engineering from King's College, London, and a $\mathrm{PhD}$ from the City University of London. 

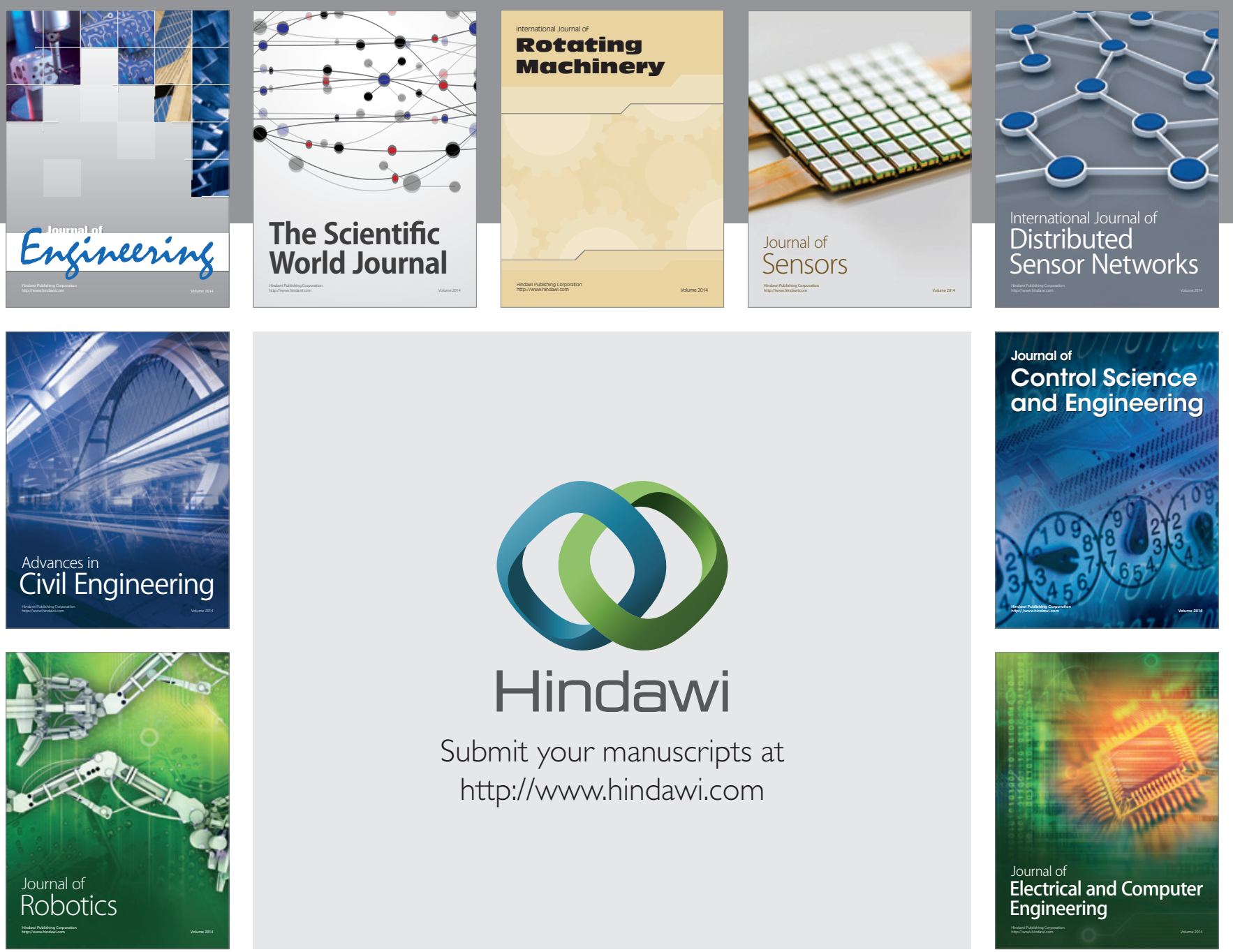

Submit your manuscripts at

http://www.hindawi.com
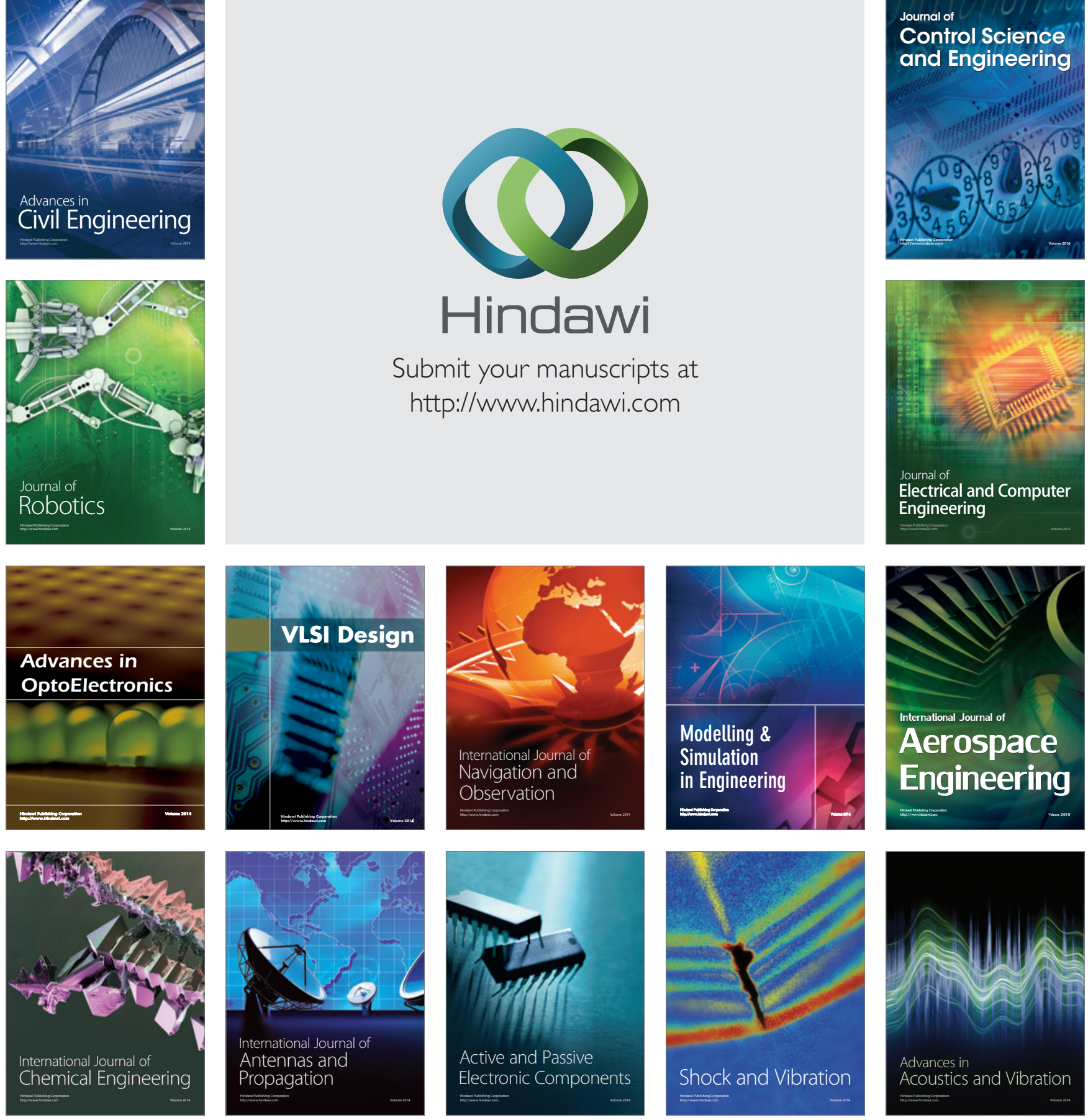